\title{
Des élites musulmanes sénégalaises dans l'action sociale : des expériences de partenariats et de solidarités
}

\section{Muriel Gomez-Perez}

\author{
(2) OpenEdition \\ Journals \\ Édition électronique \\ URL : https://journals.openedition.org/apad/4088 \\ DOI : $10.4000 /$ apad.4088 \\ ISSN : 1950-6929 \\ Éditeur \\ LIT Verlag \\ Édition imprimée \\ Date de publication : 1 août 2011

\section{Référence électronique} \\ Muriel Gomez-Perez, "Des élites musulmanes sénégalaises dans l'action sociale : des expériences de \\ partenariats et de solidarités », Bulletin de l'APAD [En ligne], 33 | 2011, mis en ligne le 22 mars 2012, \\ consulté le 21 septembre 2021. URL : http://journals.openedition.org/apad/4088 ; DOI : https:// \\ doi.org/10.4000/apad.4088
}

Ce document a été généré automatiquement le 21 septembre 2021.

Bulletin de I'APAD 


\title{
Des élites musulmanes sénégalaises dans l'action sociale : des expériences de partenariats et de solidarités
}

\author{
Muriel Gomez-Perez
}

\section{Introduction}

1 Les analystes ont eu tendance à souligner qu'à la faveur d'un contexte généralisé de suspicion envers les États au cours des années 1980, les organisations nongouvernementales (ONG) ont été perçues comme un contrepoids de l'État. Elles ont été définies comme des partenaires qui travailleraient avec les populations et proposeraient des projets en lien avec leurs volontés (Zaidi 1999 : 260-262). Cependant, la recrudescence des crises, depuis le milieu des années 1990, conduit les observateurs à relativiser le rôle des ONG, notamment dans leur capacité à permettre aux acteurs sociaux d'avoir une grande marge de manœuvre par rapport aux États (Edwards 2004 ; Kasfir 1998 ; Gellner 1994). Au-delà de ce constat global, les attributs religieux des ONG confessionnelles ont été de plus en plus perçus comme pouvant interpréter de façon adéquate les valeurs des populations (Benthall 2006, pour les ONG islamiques).

Notre réflexion ${ }^{1}$ s'inscrit dans cette perspective car elle rend compte de deux processus : d'une part, celui des reconfigurations de l'islam de plus en plus visibles dans l'espace public (Göle 2002) qui conduisent à parler de religion civile (Hann 2000; Casanova 1994) et à analyser comment les acteurs religieux participent à la construction d'un " espace public religieux » (Holder 2009; LeBlanc \& Gomez-Perez 2008; Hefner 2000); d'autre part, on assiste au réinvestissement du religieux dans la société civile qui est porteur d'un projet politique (Otayek \& Soares 2007 ; Gomez-Perez 2005) et invite à considérer les ONG confessionnelles comme des acteurs dans la régulation des sociétés (Liogier 2007 ; Renders 2002). 
3 Forts de tous ces éléments, il s'agit, en menant une analyse autour de deux cas - Jamra (association née en juin 1982 et devenue ONG en $1985^{2}$ ) et l'association Réseau national Islam Sida Éducation (née en 2002) ${ }^{3}$ - et en croisant nos données empiriques issues de trois terrains à Dakar en juin 2005 et 2008 et en mars $2010^{4}$, d'explorer comment ces deux structures, très présentes dans l'espace public, proposent des formes originales de participation citoyenne dans le cadre de la lutte contre le Sida. Nous défendons l'hypothèse selon laquelle ces initiatives mettent en évidence l'émergence de modes populaires d'action volontariste. Dans le même temps, et sans que cela ne soit contradictoire, ces participations citoyennes répondent favorablement aux programmes de prévention de l'État et des bailleurs de fonds internationaux au point que ces ONG locales deviennent des relais incontournables.

4 Nous verrons dans un premier temps comment s'articulent les relations de partenariat entre les ONG, l'État et les bailleurs de fonds internationaux et les raisons qui ont amené l'État à cibler ces ONG. Dans un deuxième temps, nous aborderons les principales conséquences de ces relations de partenariat notamment à partir de la dialectique entre les fondements éthiques de l'islam, la collaboration avec divers partenaires (État, bailleurs de fonds etc.) et l'arrimage aux programmes internationaux d'aide au développement. Enfin dans un troisième temps, nous appréhenderons en quoi ces ONG devenues des relais incontournables de la politique étatique et de la communauté internationale peuvent donner des signes d'indocilité.

\section{Partenariat entre communauté internationale, État et ONG}

5 Au Sénégal, depuis l'enregistrement du premier cas d'infection au VIH en 1986, la situation de prévalence demeure relativement faible au sein de la population globale, selon les dernières données consultables ${ }^{5}$, mais concentrée et élevée dans certains groupes tels que les prostituées, les homosexuels et les jeunes filles (Niang 2010). Tous les auteurs comme ONU Sida s'accordent pour dire que cette situation positive est due en grande partie à l'existence ancienne d'une bonne synergie entre tous les partenaires et à ce que les autorités sanitaires et politiques ont reconnu très tôt qu'elles devaient travailler avec les milieux religieux pour les campagnes de prévention. "À partir de l'année 1988, le programme national pour la lutte contre le Sida a développé des contacts intensifs avec des leaders musulmans et chrétiens afin de les impliquer dans la promotion des comportements contre la diffusion de la maladie » (Lagarde et al. 2000 : 2028). Cette prise de conscience s'est accélérée au début des années 1990, quand la «mobilisation sociale " est officiellement prônée comme stratégique : en témoigne le slogan «Les Communautés s'engagent » donné par l'OMS à la Journée Mondiale du Sida de 1992 (Delaunay 1998 : 119-120). Ainsi, la collaboration entre l'ONG Jamra et le Réseau Islam Sida a été voulue par l'État. Comme le suggère Karine Delaunay, les acteurs non gouvernementaux ont constitué des "relais" pour la diffusion des messages de prévention. Leur légitimité reposait «sur la 'proximité' dont ils pouvaient se prévaloir vis-à-vis des populations visées par lesdits messages» (Delaunay 1998: 118)6 ${ }^{6}$ Cette politique de partenariat n'est pas propre au Sénégal si l'on observe la réalité ghanéenne (Ian Gary $1996: 159$ et suiv.).

6 Ces deux structures se sont donc vues impliquées directement pour permettre la bonne marche de grandes campagnes de prévention. Ainsi, Jamra, en 1989, signe un protocole 
avec le programme national et interministériel de lutte contre le Sida ${ }^{7}$ et est partie prenante dans la création de l'Alliance des religieux en 1999 avec Sida Service, l'Association Nationale des Imams et des Oulémas du Sénégal (ANIOS) et le Programme National de Lutte contre le Sida. Jamra, depuis 2005, participe à l'exécution d'un programme sur Islam et Sida avec une approche genre en collaboration avec le Ministère de la Famille et d'un autre programme contre le paludisme financé par le Fonds des Nations Unies pour la Population (FNUAP). Quant à Ousmane Gueye, leader du Réseau National Islam Sida Éducation, il est nommé par ONU Sida comme conseiller temporaire pour créer l'alliance nationale contre le Sida. De 2003 à 2004, le Réseau National Islam Sida a mis en œuvre un programme intitulé "Coalition des religieux musulmans pour une harmonisation des messages dans une synergie d'action " en partenariat avec le Conseil National de Lutte contre le Sida (CNLS) sur financement de la Banque mondiale. Un an plus tard, un autre programme intitulé «Kiffah (lutte) contre la stigmatisation et la discrimination des Personnes vivant avec le VIH (PVVIH) » en partenariat avec le CNLS sur financement du Fonds Mondial est mis en œuvre tout comme le programme "Renforcement de l'implication des religieux musulmans dans la prévention des IST/VIH/Sida » avec les mêmes partenaires. Puis de 2005 à 2006, soit vingt ans après les premières campagnes de lutte contre le Sida, le réseau National Islam Sida sensibilise tous les leaders religieux à une approche genre en partenariat avec le Ministère de la Famille, sur financement du FNUAP8.

Plusieurs raisons expliquent que l'ONG Jamra ainsi que le Réseau soient devenus des partenaires incontournables. L'ONG Jamra est pionnière dans la lutte contre certains " fléaux sociaux » pour reprendre les termes des dirigeants de cette ONG. Elle a lancé un mensuel, Jamra, porté sur des dossiers sociaux tels que la drogue, la prostitution, la délinquance juvénile et le Sida. Elle a mis en place depuis plusieurs années un système d'accueil et d'écoute pour la réadaptation sociale des toxicomanes, des Orphelins et Enfants vulnérables (OEV) et des PVVIH, soit le geew ${ }^{9}$ qui tient à revaloriser le rôle de la famille dans un esprit de "tolérance et de respect de la conviction religieuse ». Jamra est connue pour avoir mené une campagne très médiatisée contre le réseau de prostitution à la cité universitaire Claudel. Dès ses débuts, en 1984-1985, l'association adopte la posture de ne pas faire de dinstinction entre les familles confrériques car « on misait sur les bonnes volontés $»^{10}$. Tous les leaders religieux sont alors ciblés, qu'ils soient khalifes généraux de confrérie, imâms, maîtres de daara (écoles coraniques) ou de dahira (associations religieuses) etc. Depuis 1987, l'association organise une semaine nationale anti-drogue. En 1989, lorsque l'association devient ONG, elle se donne pour tâche d'œuvrer pour la réinsertion des prostituées et des toxicomanes, pour la prévention et la prise en charge, l'organisation de groupes de parole dans les lieux de fumoir des toxicomanes. En 1995, l'ONG organise un colloque intitulé « Sida et Islam » à l'issue duquel est rédigé un guide pour les sermons des imâms. C'est aussi à la faveur du dynamisme du président Latif Gueye qu'est créee l'alliance des religieux et des experts médicaux contre l'épidémie du Sida au Sénégal en $1999^{11}$. Latif Gueye est issu d'une famille d'élite léboue de la région de Cap-Vert qui est connue pour son engagement politique et syndical ${ }^{12}$. Il est lui-même "engagé politiquement, depuis l'adolescence dans des mouvements clandestins d'obédience marxistes-léninistes, (...) fut arrêté pour la première fois par la police à l'âge de 17 ans pour activités subversives. En 1976 (...) il rejoint le professeur Cheikh Anta Diop, pour la création du « Rassemblement National Démocratique » (RND) ». (...) En 1984, (il est) rédacteur en chef du journal «Wal Fadjri $" \|^{13}$ au moment où ce journal participe pleinement et vigoureusement au débat 
islamique (Gomez-Perez 2008, 2005, 1997, 1994). Il est resté président exécutif de Jamra pendant plus de vingt ans.

Quant à Ousmane Gueye du Réseau national Islam Sida Éducation, il a probablement été choisi en raison de sa bonne connaissance des logiques et des modes de fonctionnement des milieux administratif et religieux, ce qui peut ainsi contribuer à faire de lui un véritable médiateur entre les deux. En effet, ancien conseiller pour l'enseignement de l'arabe au Ministère de l'Éducation Nationale de 1975 à 1983, il a ensuite été le secrétaire administratif et permanent de l'association des imâms du Sénégal de 1983 à 2002 sur la demande de l'ex imâm râtib de la grande mosquée de Dakar, imâm Maodo Sylla ${ }^{14}$. Cela fait plus de 25 ans qu'il anime l'émission religieuse "Lettres musulmanes " à la Radio Télévision Sénégalaise (RTS) ${ }^{15}$ ce qui lui a valu d'être une des figures remarquées du paysage médiatique et religieux.

Ces structures ont aussi été choisies car leurs responsables sont connus pour avoir tissé des relations de confiance avec l'ensemble des khalifes généraux des différentes confréries musulmanes, sans faire de distinction, ce qui est vraisemblablement un gage de réussite aux yeux de l'État et des bailleurs de fonds internationaux. Par exemple, dès la mise en place d'un protocole d'accord avec le programme de lutte contre le Sida, Jamra s'est engagée à sillonner le pays pour «informer et sensibiliser les leaders religieux musulmans sur cette nouvelle maladie et les amener à s'impliquer dans la lutte pour une meilleure prise en charge des OEV et PVVIH ${ }^{16}$. Des conférences publiques se sont tenues dans les grandes capitales confrériques telles que Touba, Tivaouane, Kaolack jusqu'en $1994^{17}$. Dans le règlement intérieur du Réseau, les khalifes généraux sont les « conseillers privilège " contactés en raison de leur influence depuis l'époque coloniale jusqu'à nos jours (Piga 2002; Cruise O'Brien et Coulon 1988; Coulon 1983; 1981), afin que les campagnes de sensibilisation puissent être relayées aisément dans les zones rurales. L'ONG Jamra s'emploie aussi à rencontrer plusieurs personnescibles telles que les représentants de l'État (préfets, sous-préfets, gouverneurs), les milieux médicaux, les communautés villageoises, les chefs de village et les imâms râtib de régions.

10 En amont, les responsables de l'ONG Jamra insistent sur le fait que la réussite de telles campagnes de sensibilisation, notamment au sujet du Sida, dépend en premier lieu de la bonne formation de ses agents. L'accent mis sur la formation contribue à donner une image de grand professionnalisme sur le terrain. Cela répond en fait au deuxième objectif de Jamra qui consiste à «créer et développer un cadre d'action et de concertation entre les différents intervenants pour une meilleure efficacité dans la lutte contre ces fléaux sociaux $»^{18}$. C'est ainsi que le «staff de Jamra a été formé sur les problématiques de la pandémie par des spécialistes tels que les professeurs Souleymane Mboup, Awa Marie Coll-Seck ${ }^{19}$ et Ibra Ndoye ${ }^{20}$. Ce dernier a également formé les imams du réseau des religieux Islam Sida ${ }^{21}$.

11 Ces collaborations entre certains médecins reconnus au Sénégal et spécialistes de la problématique du VIH et les deux sctructures, donnent plus de crédibilité à leur action de sensibilisation et de prise en charge gratuite des malades. Jamra est à cet effet en étroite relation avec l'hôpital Fann (Dakar) et tente de collaborer le plus possible avec des structures sanitaires dans les régions.

12 Mais au-delà de ce partenariat avec des experts médicaux, quelles est la dialectique entre les fondements éthiques de l'islam et la collaboration avec divers partenaires 
(État, bailleurs de fonds etc.) ? Quelles sont les grandes lignes de force du discours moralisateur des élites islamiques et les moyens utilisés pour le mettre en pratique?

\section{Vers une entente tacite entre partenaires : éthique de l'islam et actions volontaristes}

13 Prenant pour principe de départ que la religion «ne peut être qu'un facteur de développement, de cohésion, de communion et de solidarité entre les hommes ${ }^{22}$, plusieurs opuscules ont été rédigés par Jamra qui élaborent une approche islamique de certaines problématiques sociales telles que la toxicomanie, l'alcoolisme, le Sida. Ainsi, au moment de la création de l'Alliance des religieux, un guide sur les principes médicaux, coraniques et bibliques en partenariat avec l'Union européenne a été rédigé et diffusé. Depuis la mise en œuvre d'une campagne qui cible davantage les rapports hommes-femmes, Jamra a également élaboré un Guide de réflexion sur la transmission mère enfant en tenant compte des recommandations divines et des enseignements du Prophète. Plusieurs arguments sont utilisés afin de sensibiliser les populations pour que le Sida soit éradiqué ou, tout au moins, ne se propage pas. Il est fait appel à un changement de comportement de tout un chacun, en prenant pour référence le texte coranique dans lequel il est écrit : « Dieu ne change jamais l'état d'un peuple, tant que ce peuple n'a pas changé lui-même son comportement » (Sourate 13, verset 11). Le bannissement de tout acte ou propos de stigmatisation et de marginalisation des malades est aussi une constante dans les propos. Dans son document interne de présentation, Jamra souligne que « les scientifiques, qui, comme l'OMS, appellent au 'change of behaviour' trouvent là un formidable point de convergence avec les Religieux ». La formulation est édifiante dans la mesure où les principes fondamentaux de la religion sont la référence suprême à partir de laquelle doivent se rapprocher les autres milieux, notamment scientifiques et médicaux. Il s'agit d'appeler les femmes «à faire le dépistage en cas de séropositivité", d' "impliquer les hommes, les maris" afin de "faciliter la concertation » entre les époux " pour faire le test », d'« établir une négociation » entre les époux pour « l'allaitement du bébé » et d'appeler à la non-stigmatisation des mères séropositives ${ }^{23}$.

Pour autant la convergence demeure stratégique notamment lorsqu'il est question des moyens à utiliser pour combattre le Sida. Aux yeux des responsables de Jamra et du Réseau, le changement de comportement doit amener à se réconcilier avec Dieu, à moraliser sa vie au quotidien suivant les principes du Coran et de la Sunna. Fidélité et abstinence sont les maîtres mots ${ }^{24}$, tout comme il est fait appel au réconfort moral, spirituel et financier des personnes vivant avec le VIH, à la lutte contre la marginalisation et la stigmatisation des malades du Sida et à la confidentialitée ${ }^{25}$. Ce type de discours est audible au sein de toutes les communautés religieuses au Sénégal ${ }^{26}$ et en dehors de ce pays $^{27}$. Dans aucun discours, il n'est mentionné l'utilisation du préservatif à une seule exception près : dans le cas des couples « discordants » et légaux dont l'un des membres est malade ${ }^{28}$.C'est ainsi que Jenny Trinitapoli souligne à juste titre que les ONG confessionnelles «began to see AIDS as an opportunity to promote their teachings on sexual behavior and respond to unmet needs » (Trinitapoli 2006 : 255). Par ailleurs, concernant les comportements sexuels, il est rappelé qu'aucune religion n'impose le lévirat ou le sororat voire les «interdit formellement, surtout lorsqu'il y a risque de transmission ou de contamination ${ }^{29}$. 

international et celui des milieux religieux et de ces ONG ? Dans l'un des objectifs de Jamra, il est précisé qu'il s'agit de "promouvoir un partenariat (...) dans le respect de l'identité " de chacun. En d'autres termes, il y a partage des tâches dans la concertation : aux ONG de sensibiliser les populations en fonction des valeurs éthiques véhiculées par la religion; aux médecins de former les personnels des ONG pour être au fait de l'évolution des thèses scientifiques sur les causes de l'émergence du Sida et sur les facteurs de contamination. Il s'agit de donner des arguments scientifiques contredisant toute thèse qui identifie cette maladie comme une punition divine. Comme le résume très bien Bamar Gueye, c'est la fonction de médecin, qui l'amène à donner des préservatifs. Mais selon lui, pour Jamra, son «rôle, c'est de dire qu'on n'utilise pas de condoms $»^{30}$. Il importe donc de ne pas heurter la sensibilité des milieux religieux pour qu'ils participent pleinement aux campagnes mises en place.

En réalité, si les milieux religieux sont sollicités, c'est parce qu'ils ont un pouvoir d'influence sur les populations et sont légitimes pour les « éduquer » et leur enseigner ce qui est relatif au Bien ou au Mal, allant jusqu'à parler des conduites intimes dans le couple. Les sphères religieuses contrebalancent le discours médical sans le mettre en cause, condition pour être reconnues comme des relais sociaux fiables. Cette «négociation de compétences » entre milieux religieux et médicaux apparaît aux yeux des pouvoirs publics comme une stratégie pour voir les chefs religieux s'approprier ces campagnes. l'harmonie, notamment lorsqu'il s'agit de la question controversée de l'utilisation du préservatif. Tous nos interlocuteurs regrettent avec force les campagnes de distribution de préservatifs dans les villages qui sont considérées comme des agressions aux traditions des populations et incitent au libertinage et à la promiscuité sexuelle. Certains y voient même une manière de promouvoir le Sida ${ }^{31}$. Bamar Gueye rappelle que promouvoir le préservatif revient à encourager les jeunes au libertinage sexuel ${ }^{32}$.

18 Considérant la tâche que se réservent les ONG, deux objectifs sont mis en exergue : 1) développer une politique de communication afin de sensibiliser de façon permanente et récurrente les populations et contribuer à une vulgarisation des messages de façon plus efficace; et 2) mettre en place une politique de dialogue entre différentes strates de la société et différents groupes sociaux. Pour répondre à ces deux objectifs qui sont liés l'un à l'autre, ces ONG sont enclines à promouvoir de nouvelles formes de participation citoyenne dans l'espace public et de nouvelles relations entre individus. La formation des imâms $\mathrm{s}^{33}$ est au centre du dispositif. Soulignons au préalable que dès 1992, certains imâms montrent une réelle implication dans les campagnes menées par Jamra tels que l'imâm Makhtar Seck, l'imâm Lamine Senghor de Guedawaye, l'imam Sankhé de Thiaroye, l'imâm Moussa Ndour de Thiaroye et l'imâm Kanté de l'Association des Étudiants Musulmans de l'Université de Dakar ${ }^{34}$. Il est important de noter que ces imâms, issus de différentes générations, prêchent essentiellement dans des banlieues populaires de Dakar excepté pour l'imâm Kantée ${ }^{35}$.

19 L'atelier national de formation des leaders et acteurs religieux sur le plaidoyer contre la stigmatisation et la discrimination des PVVIH et des groupes vulnérables auquel nous avons assisté est dans cette perspective une action pleine d'enseignements ${ }^{36}$. Les travaux de groupes ont été effectués pour favoriser les échanges entre générations, entre hommes et femmes, entre imâms et représentants de l'ONG Jamra et réfléchir à la 
contribution des religieux dans la vulgarisation de la loi $\mathrm{VIH} / \mathrm{Sida}^{37}$ et à la réduction de la stigmatisation des PVVIH. Des exercices de simulation pour parfaire des techniques de plaidoyer auprès de différentes personnes-ressources (un décideur, un leader religieux, un chef d'entreprise, un enseignant coranique, un chef de village ou de communauté rurale) ont aussi été organisés. À travers ces travaux de groupes, plusieurs choses étaient testées: les connaissances des religieux sur les modes de transmission du Sida; leur capacité à s'adapter à différentes situations sur le terrain (en fonction des demandes des populations); leur réactivité face à celles-ci; leur capacité à mettre en adéquation leurs actions sur le terrain et leurs discours-sermons dans les mosquées pour lutter contre la stigmatisation, la peur collective face à la maladie et contre les clichés socioculturels; leurs capacités à échanger avec les populations, à les sensibiliser et à leur prêter assistance en faisant preuve de compréhension - les religieux sont également supposés conseiller les maris pour une meilleure compréhension dans les couples $^{38}$ et expliquer les méfaits de certaines traditions telles que le lévirat et le sororat - ; échanger des idées en fonction d'expériences de terrains diverses (zones urbaines/rurales, zones enclavées/de migration etc.). C'est dire l'ampleur de leur tâche dans la mesure où les religieux sont perçus comme de véritables éclaireurs dans la lutte contre le $\mathrm{VIH}^{39}$.

En repensant les techniques de communication, cette formation des imâms contribue à faire évoluer les dynamiques internes de la communauté islamique au Sénégal et à reconfigurer la place de chaque acteur religieux notamment dans la bataille menée contre le Sida. Comme le suggère Karine Delaunay, « les imams paraissent avoir été en mesure de reconquérir, face au Sida, un statut que la montée en puissance des réseaux confrériques avaient tendance à occulter dans la vie sociale; ils ont en effet pu faire valoir leur rôle de «leaders d'opinion" vis-à-vis de la communauté des fidèles, prise dans son ensemble, pour la diffusion des messages de prévention » (Delaunay 1998 : 124).

Outre la formation des imâms, d'autres relais sont exploités comme les dahira, les daara, les groupements de promotion féminine ou les associations de quartier. Ils contribuent à quadriller les zones urbaines et rurales, mais aussi à toucher l'ensemble des milieux sociaux et à intégrer davantage les femmes dans les actions. Le dialogue demeure un point essentiel dans le dispositif des actions menées par ces deux structures ce qui explique la tenue de séminaires de formation, de colloques, de causeries dans les quartiers et l'animation d'émissions radiophoniques. Par exemple, en 1995, le colloque Sida et Islam sert à collecter les suggestions des Religieux "pour une meilleure vulgarisation des messages liés aux IST/VIH/Sida ». En 1997, le colloque Sida et Religion à Dakar a permis de contribuer au dialogue entre catholiques et musulmans et de voir la mise en place deux ans plus tard de l'Alliance des Religieux Internationale avec comme conseillers spirituels l'imâm râtib de Dakar et le Cardinal de Dakar. L'émission "Deukendo Diamathia Guene " ${ }^{40}$ animée par l'imâm Moussa Gueye à la radio Dunya, lequel a aussi créé des groupements du même nom dans le quartier Parcelles Assainies ${ }^{41}$ à Dakar, illustre bien la volonté non seulement de mettre en pratique des stratégies de dialogue entre aînés et cadets ${ }^{42}$ mais aussi d'instaurer des relais à travers des associations de quartiers, dans les mosquées. Cette initiative a d'ailleurs été étendue à certaines régions telles que celles de Dagana, Louga, Saint-Louis, Thiès et Rufisque. Jamra utilise, à ce titre, les expertises de ses membres fidèles tels que l'imâm Moussa Gueye, qui depuis 20 ans anime des émissions religieuses à des différentes radios au cours desquelles il appelle à avoir un « comportement correct $»^{43}$. Le fait que cet imâm 
soit connu pour ses conférences diffusées à la radio et ait initié la création d'un réseau associatif dans la banlieue dakaroise conduit Jamra à initier avec lui et à sa demande des actions ponctuelles de sensibilisation auprès des jeunes. Lors d'une action de sensibilisation des parents d'élèves dans une école primaire du quartier Rafat II à Rufisque $^{44}$, il s'agissait par exemple de rappeler à l'assistance (essentiellement composée de jeunes mères et d'enseignantes) les modes de transmission du Sida et le rôle central que doivent jouer les femmes et les parents dans la non prolifération de cette maladie. Ainsi, les intervenants (Bamar Gueye et Ousmane Gueye) ont insisté sur le fait que tout parent doit veiller à surveiller ses enfants, à les éduquer en leur transmettant les principes éthiques de la religion et en faisant connaitre le licite et l'illicite. Ils ont aussi insisté sur l'abstinence, la fidélité, la non stigmatisation mais aussi sur l'importance de la repentance ${ }^{45}$ et sur le fait de veiller à ce que les jeunes n'aient pas des relations sexuelles précoces et d'inciter les personnes à faire le dépistage prénuptial car il est gratuit, volontaire et anonyme. À l'issue de la causerie, place était donnée aux questions de l'assistance. En définitive, ces différents relais montrent une « reconnaissance des dynamiques populaires et de leur légitimité et s'appuient sur la participation des habitants et le contrôle des citoyens » (Revel \& Roca 1998 : 92).

L'ensemble de ces actions volontaristes et participatives contribuent en définitive à des reconfigurations de l'islam dans la mesure où ces deux ONGs sont des «lieux de formation à la participation à l'action publique » ('Abd Al-Ghaffar Shukr 2004). Elles participent à une restructuration de la société civile en se fixant pour objectif de «maîtris(er) sa destinée en faisant l'histoire ${ }^{46}$ ou/et « d'être des artisans d'un futur choisi $\aleph^{47}$. Cette participation à l'action publique prend à quelques reprises des contours plus politiques qui conduisent à s'interroger sur le rôle des ONG dans le débat politique et ses conséquences sur les relations de modus vivendi entre État sénégalais et ONG.

\section{Vers une stratégie de lobbying}

Bien que les deux structures soient des relais sociaux incontournables pour l'État, celles-ci montrent néanmoins une volonté de garder une distance raisonnable avec l'État, une autonomie d'action et de pensée et une volonté de se préserver une marge de manœuvre pour épingler avec virulence certaines mesures gouvernementales. Dans cette perspective, Bamar Gueye, à plusieurs reprises, souligne sa volonté d'«être autonome » et de ne "pas prêter le flanc pour que les activités d'ONG ne soient pas suspendues par l'État $»^{48}$. Il va encore plus loin en revendiquant la nécessité de rester apolitique, comme les statuts le stipulent, «car des membres de Jamra ne sont pas du $\mathrm{RDS} »^{49}$. Ainsi l'apolitisme est brandi pour ne pas tomber dans le piège des luttes partisanes. Selon les propos de Bamar Gueye, cet apolitisme affiché a toujours fait l'objet de vives discussions avec son frère Latif qui, contre l'avis de Bamar, aurait souhaité tenir des réunions politiques dans les locaux de Jamra. Pour Bamar, il s'agit ne pas jeter de discrédit sur l'action de l'ONG, pour que ne s'instaure pas une confusion dans l'opinion publique sur l'action sociale menée par l'ONG et sur l'action politique menée par son président. Il est en effet symptomatique que parallèlement à ce refus de position partisane, Bamar Gueye rappelle à loisir que l'ONG a acquis sa réputation sur le fait d'être constante dans sa lutte contre les fléaux sociaux. Par ailleurs, le fait que Latif Gueye, au faîte de sa carrière ${ }^{50}$, ait subi des démêlées judiciaires et ait été mis en détention préventive pendant 14 mois (juin 2003-août 2004) (11 $^{1}$ tend à donner raison à 
son frère Bamar de garder ses distances avec le monde politique pour ne pas être victime de certaines jalousies au sein du gouvernement. L'actualité lui donne apparemment encore raison dans la mesure où le RDS vient de connaître une scission ${ }^{52}$.

La distance avec l'État demeure aussi perceptible en interrogeant nos interlocuteurs sur leurs relations avec le Conseil des ONG d'appui au Développement au Sénégal $(\text { CONGAD })^{53}$, auquel ils ne souhaitent pas être intégrés. À mots couverts, ils expriment une certaine distance vis-à-vis du CONGAD, préférant ne pas être affiliés à cette structure pour ne pas avoir les pieds et poings liés avec l'État. Comme le souligne Bamar Gueye, " on veut rester indépendant. Là-bas, il y a quelques restrictions pour limiter nos attaques et nos champs d'action ${ }^{54}$. À la fin des années 1990, Marleen Renders constatait déjà cette méfiance : «one CONGAD official declared that 'Islamic NGOs are for several reasons rather marginalised within the organisation. The relation between the predominantly secular Senegalese and international membership of CONGAD and the 'Islamic' NGOs is somewhat uneasy. The principles of CONGAD do not directly appeal to them, although de facto they would not have a problem adhering to them. Another reason for the small number of 'Islamic' member NGOs is reportedly obstruction of such NGOs by the state authorities for reasons of national security" (Renders 2002: 63).

L'apolitisme affiché de Jamra dans ses statuts et la volonté de laisser une marge de manœuvre vis-à-vis de l'État ne signifient pas pour autant que cette ONG ne participe pas au jeu politique. À plusieurs reprises, Jamra s'est placée sur le devant de la scène pour alerter à la fois l'opinion publique et les autorités devant des faits de société. Elle a investi largement l'espace public et exerce une certaine surenchère verbale par ses appels virulents en faveur de la moralisation des mœurs, contre la prostitution et les actes de violence sur les jeunes filles (viols et incestes) et par ses marches de protestation contre la diffusion d' «images obscènes» dans les médias ou contre la libération d'homosexuels par la justice. À ce titre, Marleen Renders souligne que «these organizations (Islamic associations and NGOs) are not political movements stricto sensu, therefore are not directly involved in the 'high politics' of the Senegalese polity. Yet, individual members sometimes are, and through their activities (or even activism) Islamic associations and individual Muslims do have a relevant political impact" (Renders 2002: 62).

Ainsi, Jamra, par ses déclarations et actions, tente de faire pression sur l'État pour le faire fléchir et obtenir des résultats à la hauteur de ses espérances. En 2002, Jamra prend l'initiative d'organiser une marche de protestation contre des images " obscènes » jusqu'au Ministère de la Communication au moment où Latif Gueye était diplomate et avait rang de ministre itinérant dans l'ONG « Afrique Aide Afrique » (AAA) après sa désignation par le président de la République, Abdoulaye Wade ${ }^{55}$. Cette manifestation n'a pas vraiment eu d'effets concrets dans les médias. Le même constat a pu être dressé l'année suivante lorsque Jamra a participé aux journées parlementaires pour sensibiliser les députés sur la nécessité d'une révision des textes législatifs concernant le Sida. Cette action a encore donné peu de résultats aux dires de Bamar Gueye. Dans le même sens, Latif Gueye dirige en tant que vice-président un Réseau parlementaire de protection contre les violences et les abus faits aux enfants en avril 2009 pour tenter de prendre en charge psychologiquement et socialement les victimes et de « faire en sorte que l'impunité ne soit plus de mise, en engageant notamment des 
poursuites en justice en faisant en sorte que la législation en ce domaine soit renforcée $»^{56}$. Les avancées se font encore attendre.

En revanche, l'action menée contre les trafiquants de drogue a connu davantage de succès. En 2007, Latif Gueye use de son influence de député pour faire adopter la loi qui criminalise le trafic de drogue. Toutefois, c'est davantage sont statut de député qui permet cette avancée plutôt que son action au sein de l'ONG Jamra.

En outre, Jamra mène depuis plusieurs années une lutte contre les homosexuels et somme l'État de prendre position dans ce débat. Suite à la publication dans un magazine people de février de 2008, d'un reportage sur un "présumé mariage entre deux hommes ", abondamment illustré de photographies d'hommes au visage masqué ${ }^{57}$, les milieux religieux dont le collectif des imâms ${ }^{58}$ et le collectif des associations islamiques ${ }^{59}$ se font les chantres de l'ordre moral en lançant des attaques virulentes et très médiatisées à l'encontre de l'État qui est taxé d'être faible et complice devant cette orientation sexuelle. L'arrestation de dix personnes soupçonnées d'avoir adressé des menaces de mort au directeur de publication puis leur libération met le feu aux poudres : les collectifs appellent à une marche de protestation, ensuite interdite par les autorités pour préserver l'ordre public. Un an plus tard, le 23 avril 2009, suite à la libération d'homosexuels pour vice de forme, Jamra réitère ses positions nettement hostiles envers les homosexuels en faisant une déclaration tonitruante intitulée " Libération des homos partouzards de Mbao : Victoire (provisoire) du mensonge et de l'hypocrisie !» dans laquelle l'ONG réitère sa position «à bannir sans équivoque cette pratique (l'homosexualité) contre-nature ", « bestiale et avilissante ", « dégradante » et "condamnée par le Coran ("Les Murailles», sourate 7) ou l'Ancien Testament (18.22) ». Au-delà de cette volonté de marquer son opposition à la libération des homosexuels, il s'agit pour Jamra de rappeler une nouvelle fois à l'opinion publique que l'homosexualité est une "déviance " et non une "simple orientation sexuelle», et qu'elle est un "vecteurde propagation du Sida ». Elle entend donc «exiger une plus grande rigueur de l'État dans la protection morale de notre jeunesse et contre les agressions multiformes dont l'islam est depuis quelque temps l'objet dans notre pays, fort de ses $95 \%$ d'adeptes ». Après avoir créé un Front islamique pour la défense des valeurs éthiques ${ }^{60}$ contre l'homosexualité, Jamra entend faire pression sur l'État sénégalais ${ }^{61}$, afin que ne soit pas signé le projet de convention pour la dépénalisation du délit d'homosexualité, déposé à l'ONU par le biais deRama Yade, secrétaire d'État aux droits de l'Homme en France à l'époque. Le bureau exécutif de Jamra ${ }^{62}$ ne ménage pas ses efforts dans ce dossier au point de «profiter des éditions 2009 du Gamou et du Magal, pour demander à Sérigne Mouhamadou Lamine Bara Mbacké et Sérigne Mouhamadou Mansour Sy de prier pour Maître Wade, pour qu'il ne tombe pas dans le piège de ces affairistes, qui de plus en plus font de la problématique de l'homosexualité un fond de commerce $»^{63}$. Finalement le 17 mai 2009, quand la convention est rejetée "sans équivoque", ils félicitent les chefs religieux maraboutiques et le Premier ministre, Souleymane Ndéné Ndiaye.

Parallèlement à ces questions sociales, Jamra s'engage plus frontalement dans le débat politique à l'occasion de la construction du Monument de la Renaissance en s'opposant très clairement à Abdoulaye Wade. Dans ce cas de figure, Bamar Gueye ne s'exprime plus en tant que directeur exécutif de l'ONG Jamra mais en tant que secrétaire exécutif du collectif des associations islamiques et des imams au Sénégal et l'imâm Massamba Diop en tant que président de l'organisation islamique Jamra laquelle est une instance à 
dimension politique et prédicative ${ }^{64}$. En décembre 2009, à la suite de la construction du Monument de la Renaissance, Bamar Gueye fustige avec force cette initiative du président de la République Abdoulaye Wade en déclarant «au moment où la société sénégalaise traverse une crise multidimensionnelle économique et sociale sans précédent, il est incompréhensible de dépenser des milliards pour édifier une statue symbolisant la culture de la nudité et de la décadence " et en ajoutant que " cette démarche constitue une preuve tangible qu'il y a une volonté manifeste de vouloir modifier ou corrompre la foi du peuple Sénégalais et ses principes fondamentaux ». Sur cette même lancée, il a appelé les autorités étatiques "à respecter les convictions religieuses et spécifiquement islamiques des Sénégalais " lors d'une conférence de presse dans la mosquée " inachevée » de l'aéroport après que le président les aient traités d'ignorants ${ }^{65}$. Ce lieu de rassemblement n'est d'ailleurs pas pris au hasard dans la mesure où il a symbolisé un des hauts lieux de la contestation islamique sous Léopold Sédar Senghor et Abdou Diouf (Gomez-Perez 2009). Ces exemples conduisent à penser que Jamra s'inscrit dans la thèse que la civilité «est à la base d'une culture de la délibération publique, de l'intervention et de la participation active des sujets publics dans le champ politique » (Fall \& Diouf 2000).

\section{Conclusion}

Ces cas sénégalais illustrent clairement l'ambivalence des relations entre ONG et État qui oscille entre complémentarité et concurrence. En effet, l'État comme les bailleurs de fonds internationaux considèrent les ONG comme des relais indispensables et incontournables pour sensibiliser les populations dans le cadre de la lutte contre le Sida. Leurs responsables sont connus du monde politique ou ont fait carrière dans la haute administration. Ils ont su gagner la confiance des divers milieux confrériques par leur capacité à se distancier de toute activité politicienne. Leur crédibilité auprès des populations et des partenaires politiques et internationaux, repose aussi sur leur partenariat étroit avec des experts médicaux.

31 Forts de leurs acquis, Jamra et le Réseau construisent un discours basé sur les fondements éthiques de l'islam et se réfèrent largement aux sourates du Coran pour convaincre les populations de changer de comportement. En cela, elles signifient la préséance du religieux sur tout autre référent tout en invitant à un partenariat responsable avec les milieux médicaux pour aboutir à une «négociation de compétences». La clé de voûte de tout ce dispositif est de mettre l'accent sur la formation des imâms qui sont considérés comme les meilleurs guides et passeurs d'idées pour réduire la discrimination des personnes porteuses de la maladie du Sida. Ainsi les ONG confessionnelles contribuent à faire émerger des processus socioculturels particuliers: la reconfiguration interne des milieux religieux par la prééminence des imâms, une nouvelle mise en place de délégation de pouvoir par la multiplication des relais sociaux à travers la diversité du tissu associatif, ou encore l'organisation de causeries, de conférences et la participation à des émissions radiophoniques. Toutes ces actions conduisent à rendre active la participation citoyenne dans l'espace public.

De plus, bien qu'étant des relais sociaux pour l'État, ces organisations religieuses revendiquent leur apolitisme dans leurs statuts, ce qui ne les empêche pas de participer pleinement au débat politique en tant que membre de la société civile au point d'être virulentes face à certaines mesures de l'État. L'ambivalence des relations entre ONG et 
État est dès lors indéniable et montre une imbrication implicite entre eux. Comme l'indique Akbar Zaidi : «Both the state and NGOs, in all likelihood, need each other to continue their symbiotic relationship, based equally on antagonism and cooperation » (Zaidi 1999 : 263). En définitive, les ONG jouent le double rôle de médiateur sociopolitique et d'activateur du débat public sous toutes ses formes.

\section{BIBLIOGRAPHIE}

ABD AL-GHAFFAR SHUKR, 2004. "Les ONG islamiques et la démocratie en Egypte », in S. Ben Néfissa, N. Abd al-Fattah, S. Hanafi. C. Milani, ONG et gouvernance dans le monde arabe, Karthala/ CEDEJ : 197-211.

BAYART, J-F, 1993. The State in Africa:The Politics of the Belly, New York, Longman Publishers. BEBBINGTON, A. \& RIDDELL, R., 1995. "The direct funding of Southern NGOs by donors: new agendas and old problems”, Journal of International Development, 7 (6): 879-893.

BENEDETTI, C., 2006. "Islamic and Christian inspired Relief NGOs : between tactical collaboration and strategic diffidence?", Journal of International Development, 18: 849-859.

BENTHALL, J., 2006. “Islamic aid in a North Malian Enclave”, Anthropology Today 22 (4): 19-21

BRATTON, M., 1989. "The politics of government-NGO relations in Africa”, World Development, 17 (4): 569-587.

CASANOVA, J., 1994. Public Religion in the Modern World, Chicago University Press.

CHABAL, P., 1992. Power in Africa, New York, St Martin's.

CLARK, J., 1991. Democratizing Development, Earthscan, London.

CODOU, B., « Sénégal : « Homophobie et manipulation politique de l'islam », Women Living Under Muslim Laws Networkers.

COMAROFF, J. \& COMAROFF, J., eds., 1999. Civil society and the Political Imagination in Africa. Critical perspectives, Chicago and London, The University of Chicago Press.

COULON, C., 1983. Les musulmans et le pouvoir en Afrique noire, Paris, Karthala.

COULON, C., 1981. Le marabout et le Prince. Islam et pouvoir au Sénégal, Paris, Pedone.

CRUISE O'BRIEN, D. B. \& COULON, C., 1988. Charisma and Brotherhood in African Islam, Oxford, Clarendon Press.

DELAUNAY, K., 1998. « Des ONG et des associations: concurrences et dépendances sur un "marché du Sida" emergent. Cas ivoirien et sénégalais », in Deler, J-P., Fauré, Y.A., Piveteau, A. et Roca, P.J. (dir.), ONG et développement. Société, économie, politique, Paris, Karthala : 115-141.

DELER, J.-P. et al. dir. 1998. ONG et développement. Société, économie, politique, Paris, Karthala.

DENIS, Ph. \& BECKER, Ch. (dir.), 2006. L'épidémie du Sida en Afrique subsaharienne, Paris, Karthala.

DIOP, M. C. \& DIOUF, M., 1990. Le Sénégal sous Abdou Diouf, Paris, Karthala. 
EDWARDS, M., 2004. Civil Society. Cambridge, England, Polity Press.

EDWARDS, M. and HULME, D., 1995a, "NGO Performance and accountability in the post-cold war world”, Journal of International Development, 7, 6: 849-856.

EDWARDS, M. \& HULME, D., 1995b. Non-governmental organizations-performance and accountability, Earthscan, London.

FALL, A.S. \& DIOUF, M., 2000. « La société civile en Afrique de l'ouest : configurations et défis », Solidarités, vol. 31, n.2 : n.p.

FOWLER, A., 1993. "Non-governmental organizations as agents of democratization: an African perspective", Journal of International Development, 5, 3: 325-339.

FOWLER, A., 1991. 'The role of NGOs in changing state-society relations: perspectives from Eastern and Southern Africa', Development Policy Review, 9.

GARY, I., 1996. 'Co-operation or Co-optation: NGOs and the Ghanaian State during Structural Adjustment', Review of African Political Economy, Vol. 23, No. 68: 149-168.

GELLNER, E. 1994. Conditions of Liberty : civil society and its rivals, New York, Allen Lane/Penguin Press.

GÖLE, N., 2002. "Islam in Public: New visibilities and New imaginaries", Public Culture, 14, (1): 173-190.

GOMEZ-PEREZ, M., 2009. «Autour de mosquées à Ouagadougou et à Dakar: lieux de sociabilité et reconfiguration des communautés musulmanes», L. Fourchard, O. Goerg et M. Gomez-Perez (éd.), Lieux de sociabilité urbaine en Afrique, Paris, L'Harmattan : 405-433.

GOMEZ-PEREZ, M. 2008 (Spring). «The Association des Étudiants Musulmans de l'Université de Dakar (AEMUD). Between the Local and the Global: An Analysis of Discourse», Africa Today, M. N. LeBlanc et B. F. Soares (dir.), «Muslim West Africa in the Age of Neoliberalism», Thematic Issue, Vol. 54, Issue 3: 95-117.

GOMEZ-PEREZ, M., 2005. L'islam politique au sud du Sahara. Identités, discours et enjeux, Paris, Karthala.

GOMEZ-PEREZ, M., 1997. Une histoire des associations islamiques sénégalaises (Saint-Louis, Dakar, Thiès) : itinéraires, stratégies et prises de parole (1930-1993), doctorat de troisième cycle d'histoire à Paris 7 Denis Diderot.

GOMEZ-PEREZ, M., 1994. «L'Islamisme à Dakar : d'un contrôle social total à une culture du pouvoir ?», Afrika Spectrum, n²9 : 79-98.

HANN, C. 2000. « Problems with the (De)Privatization of Religion », Anthropology Today, 16 (6) : 14-20.

HARBERSON, J., ROTHCHILD, D. \& CHAZAN, N. (eds), 1994. Civil Society and the State in Africa, Lynne Reimer, Boulder, Co.

HEALEY, J. and ROBINSON, M., 1992. Democracy, governance and economic policy: sub-Saharan Africa in comparative perspective, ODI, London.

HEFNER, R. W., 2000. Civil Islam: Muslims and Democratization in Indonesia, Princeton, Princeton University Press.

HOLDER, G., 2009. L'Islam, nouvel espace public en Afrique, Paris, Karthala. 
KASFIR, N. 1998. "The Conventional Notion of Civil Society: A Critique”, in Nelson KASFIR (dir.), Civil Society and Democracy in Africa: Critical perspectives. London, Frank Cass: 1-20.

KELSALL, T., 2001. "Donors, NGOs and the State", in Ondine Barrow and Michael Jennings, The Charitable Impulse. NGOs and Development in East and North-East Africa, James Currey, Oxford and Kumarian Press: 133-148.

KHILNANI, S., 2001. « La 'société civile ', une résurgence », Critique internationale, 10 : 38-50.

LAGARDEet alii, 2000. "Religion and protective behaviours towards AIDS in rural Senegal", AIDS, Vol. $14, n^{\circ} 13: 2027-2033$.

LEBLANC, M.N. \& GOMEZ-PEREZ, M., 2008. “Jeunes musulmans et citoyenneté culturelle : retour sur des expériences de recherche en Afrique de l'Ouest francophone", Sociologie et Société, Calvès et Marcoux (dir.), 39 (2) : 39-59.

LEWIS, P., 1992. "Political Transition and the Dilemma of Civil Society in Africa", Journal of International Affairs, 27: 31-54.

LIOGIER, R., 2007. «L'ONG, agent institutionnel optimal du champ religieux individuo-globalisé », Duriez, B. et alii, Les ONG confessionnelles. Religion et action internationale, Paris, L'Harmattan : 263-280.

LOM, M. M., 2001. « Le Sénégal, un modèle de réussite. La mobilisation précoce et l'engagement politique one enrayé la progression du VIH », Afrique Relance, ONU, vol. 15, nos 1-2 : 24.

MERCER, C., 1999. "Reconceptualizing state-society relations in Tanzania: are NGOs 'making a difference'?", Area, 31, $3: 247-258$.

MICHAEL, S., 2004. "The power of local NGOs in Senegal”, in Michael, S., Undermining Development. The absence of power among local NGO in Africa, James Currey, Indiana University Press: 91-111.

MWAURA, P., 1999. « Innover dans la lutte contre le Sida. L'Ouganda et le Sénégal prouvent qu'il est possible de réduire les taux de contamination ", Afrique Relance ONU, vol. 12, no 4 : n.p.

ORUBULOYE, I.O., CALDWELL, John \& CALDWELL, Pat, 1993. "The role of religious leaders in changing sexual behaviour in Southwest Nigeria in an Era of AIDS", Health Transition Review, 3 : 93-104.

OTAYEK, R., 2002. «Société civile en Afrique: de l'utilité du regard décentré», Revue internationale de politique comparée, 9 (2) : 193-212.

OTAYEK, R. \& SOARES, B., 2007. Islam and Muslim Politics in Africa, Londres, Palgrave Macmillan.

PFEIFFER, J., 2004a. « Civil Society, NGOs and the holy Spirit in Mozambique », Human organization, Vol. 63 , No. $3: 359-372$.

PFEIFFER, J., 2004b. « Social Marketing, Pentecostalism, and Structural Adjustment in Mozambique : a Clash of AIDS Prevention messages ", Medical Anthropology Quarterly, 18 (1) : 77-103.

PIGA, A., 2002. Dakar et les ordres soufis, Paris, L'Harmattan.

RENDERS, M., 2002. « An ambiguous adventure: Muslim organisations and the discourse of 'development' in Senegal », Journal of religion in Africa, 32.1: 61-82.

RÉSEAU ISLAM ET POPULATION, 1996. La déclaration de politique de population à la lumière des enseignements islamiques, République du Sénégal/FUNUAP. 
REVEL, M \& ROCA, P-J., 1998. " Les ONG et la question du changement. Jusqu'où les ONG changeront-elles? Jusqu'où pourront-elles changer ? », in Deler, J-P., Fauré, Y.A., Piveteau, A. et Roca, P.J. (dir.), ONG et développement. Société, économie, politique,Paris, Karthala : 89-103.

SECHER MARCUSSEN, H., 1998. «Les ONG et la construction de la société civile dans les pays en développement », in Deler, J-P., Fauré, Y.A., Piveteau, A. et Roca, P.J. (dir.), ONG et développement. Société, économie, politique, Paris,Karthala : 573-597.

SOW DIA, H., 2007. État et société civile au Sénégal. Thèse de doctorat, Frankfurt am Main, Lang, Coll. « Berliner Studien zur Politik in Afrika », 14.

TRINITAPOLI, J., 2006. «Religious responses to AIDS in Sub-Saharan Africa : An Examination of religious Congregations in Rural Malawi », Review of Religious Research, Vol. 47, No.3: 253-270.

VUARIN, R., 1990. “L'enjeu de la misère pour l'islam sénégalais ", Revue Tiers Monde, XXXI, numéro 123: 601-621.

ZAIDI, S. AKBAR, 1999. 'NGO failure and the need to bring back the state', Journal of International Development, March-April, 11 (2): 259-271.

\section{NOTES}

1. Je tiens à remercier chaleureusement Seydi Ibrahima Ly, étudiant en maîtrise au département d'histoire de Dakar, pour son aide dans notre recherche lors de notre séjour à Dakar entre février et mars 2010.

2. Les ONG confessionnelles sont souvent des prolongements des associations religieuses dans la mesure où un agrément en tant qu'ONG ne peut être délivré par le Ministère du Développement Social et de la Solidarité Nationale qu'au terme, notamment, de deux ans d'activités en tant qu'association.

3. C'est un regroupement d'ONG et d'associations : Association des imâms et oulémas de Thiès, Jamra, Union des imams et prédicateurs du Sénégal, Mouvement omarien pour l'humanisme, Union pour le progrès islamique au Sénégal (UPIS), Association préventive de lutte contre la drogue, la toxicomanie et le VIH, Union culturelle et sportive des écoles franco-arabes et daaras de Rufisque, Association sénégalaise des musulmans pour l'action sociale, daara Cheikh Ahmed Tidiane Chérif, Association pour le développement de l'enfance, association islamique Sayda Soumaya, dahira Syda Khadidja, Association sénégalaise pour le développement de l'enseignement islamique, Association pour la lutte contre la mendicité des handicapés et des talibés, Association des jeunes imâms de Mbour... (entretien avec le dirigeant Ousmane Gueye, à son bureau, Institut islamique, Dakar, le 6 juin 2008).

4. Nous avons mené une série d'entretiens semi-directifs d'une part, avec les responsables de ces deux structures en les interrogeant sur leurs itinéraires, leurs actions passées, présentes et à venir, sur la dialectique entre le Politique, les bailleurs de fonds internationaux et leurs propres convictions religieuses et d'autre part, avec certains de leurs collaborateurs qui sont imâms afin d'identifier leurs itinéraires et les raisons de leur participation à certaines activités de ces structures. Parallèlement à cela, nous avons mené des observations participantes lors de certaines activités telles que la manifestation annuelle organisée par Jamra sur la lutte contre la drogue et le sida et la remise des prix à l'issue du concours national de poèmes au théâtre national Daniel Sorano à Dakar, une causerie organisée conjointement par les deux structures dans une école à Rufisque pour sensibiliser les populations sur le sida et l'atelier national de formation des leaders et acteurs religieux sur le plaidoyer contre la stigmatisation et la 
discrimination des personnes vivant avec le VIH et des groupes vulnérables tenu dans les murs de l'Institut islamique à Dakar.

5. Le taux de prévalence est de 0,7\% (source EDS IV-2005, quatrième enquête démographique de santé). L'Ouganda est, avec le Sénégal, considéré comme un pays de référence (Mwaura 1999).

6. L'auteur précise que les Plans Nationaux de Luttre contre le Sida (PNLS) «ont eu pour fonction la programmation et la coordination des actions au niveau des États, constituant, parallèlement, les relais officiellement obligés d'acheminement et de répartition de l'aide internationale consacrée à la lutte contre le Sida » (Delaunay et al. 1998 : 117).

7. Entretien avec Aïssatou Mbaye, membre du bureau, s'occupant de la coordination, au siège de Jamra, Dakar, le 4 juin 2008.

8. Cette problématique paraît tardive dans la mesure où les programmes de lutte contre le Sida ont débuté dès 1986. En fait, cette problématique était prise en compte dans les actions par Jamra notamment depuis plusieurs années aux dires de Bamar Gueye, directeur exécutif de l'ONG Jamra.

9. Signifie «cercle» en wolof qui "intègre dans le même processus l'accueil, l'écoute et la réadaptation sociale, Latif Gueye, Le « Geew », Les éditions Djamra, mars 1988, n.p.

10. Entretien avec Aïssatou Mbaye, au siège de Jamra, Dakar, le 4 juin 2008.

11. Latif Gueye en était le secrétaire général et Émile Daly Diouf, de confession chrétienne, en était le secrétaire général adjoint et le président de Sida Service.

12. Son père Abbas Gueye est député du Sénégal de 1951 à 1955. Aux côtés de Léopold Sédar Senghor et de Mamadou Dia, il quitta le parti socialiste sénégalais, dirigé par Lamine Gueye et fonda le Bloc démocratique sénégalais (BDS). Il fut candidat à Dakar aux élections à la deuxième Assemblée législative et figura en seconde position sur la liste du BDS conduite par Léopold Sédar Senghor, député sortant. Élu et inscrit au groupe des Indépendants d'outre-mer, il fut membre de la Commission du travail et de la sécurité sociale pendant toute la durée de la législature. En novembre 1952, à la discussion du projet de loi instituant un code du travail dans les territoires d'outre-mer, il intervint notamment sur le travail forcé, la durée légale du travail, les pouvoirs des inspecteurs du travail. De retour à Dakar, Abbas Gueye devint, en 1960, secrétaire général de l'Union générale autonome, seul syndicat autorisé après l'indépendance du Sénégal.

13. Service communication du RDS, le lundi 6 avril 2009.

14. Entretien avec Ousmane Gueye, à son bureau, Institut islamique, Dakar, le 11 juin 2008. Il faut savoir que Ousmane Gueye et Maodo Sylla se sont connus tout jeune, dès l'âge de 7 ans, puisqu'ils fréquentaient la même daara à Bargny dans les années 1940.

15. La première émission télévisée de ce type diffusée à la RTS. C'est Sokhna Dieng qui avait appelé Ousmane Gueye pour animer cette émission lorsqu'il dirigeait l'association des imâms (Entretien avec Ousmane Gueye, à son bureau, à Dakar, le 6 juin 2008).

16. Entretien avec Aïssatou Mbaye, au siège de Jamra, Dakar, le 4 juin 2008.

17. Entretien avec Aïssatou Mbaye, au siège de Jamra, Dakar, le 4 juin 2008.

18. Document interne de présentation de Jamra.

19. Spécialiste dans la recherche sur le VIH/Sida, elle dirigea, à Genève, le département Politique, stratégie et recherche de l'Onusida entre 1996 et 2001 puis fut nommée Ministre de la santé entre 2001 et 2003.

20. Entretien avec Aïssatou Mbaye, au siège de Jamra, Dakar, le 4 juin 2008. Ibra Ndoye est le coordonnateur du PNLS et responsable d'un centre de traitement et de recherche sur le Sida à l'hôpital Fann de Dakar.

21. Entretien avec Ousmane Gueye, dans son bureau, Dakar, le 6 juin 2008.

22. Document interne de présentation de Jamra.

23. Guide de réflexion sur la transmission mère enfant en tenant compte des recommandations divines et des enseignements du Prophète, p. 4. 
24. Alliance des religieux et des experts médicaux dans la réponse à l'épidémie du Sida au Sénégal, Les principes médicaux, coraniques et bibliques que tout croyant doit lire, savoir et appliquer, $\mathrm{p}$. 8. Ce document a été élaboré conjointement par le Comité Sida, l'ONG Jamra, l'ONG Sida Service et l'ANIOS. Il est le résultat de : la mise en place en 1999 de l'Alliance de religieux Internationale à Dakar qui souhaite établir une synergie entre toutes les communautés religieuses au Sénégal ; l'organisation, entre 1999 et 2003, a fait des tournées auprès des leaders religieux de toutes confessions, auprès des autorités administratives et médicales et a organisé des ateliers de formation pour sensibiliser ces leaders à la question du Sida. L'éloge de la chasteté et de la fidélité tout comme l'interdiction de la fornication et de la turpitude associée à l'homosexualité sont aussi soulignées dans le Guide islam et Sida. Recueil de sermons et conférences, n.p. La polygamie est considérée comme pouvant «être un frein à l'anarchie sexuelle et donc à la propagation du Sida », op. cit. Réfréner les relations sexuelles extras-conjugales est un argument souvent utilisé (Orubuloye et alii 1993).

25. Il est souligné toutefois que "dans un couple légal, il est souhaitable que les conjoints s'informent réciproquement pour éviter la propagation au sein de la famille», Alliance des religieux et des experts médicaux dans la réponse à l'épidémie du Sida au Sénégal, Les principes médicaux, coraniques et bibliques que tout croyant doit lire, savoir et appliquer, p. 13.

26. Jamra travaille avec Sida Service, une ONG chrétienne, qui use du même discours, "Paul Sagna, secrétaire exécutif de Sida service, reconnaît que son association ne rejette pas l'utilisation du préservatif comme moyen de prévention mais conseille plutôt « l'abstinence et la fidélité » (Mika 2001 : 24).

27. Pfeiffer (2004b) l'a souligné aussi au Mozambique.

28. Entretien avec Ousmane Gueye, dans son bureau, Dakar, le 6 juin 2008 et entretien avec Bamar Gueye, dans son bureau, Dakar, le 1er mars 2010.

29. Alliance des religieux et des experts médicaux dans la réponse à l'épidémie du Sida au Sénégal, Les principes médicaux, coraniques et bibliques que tout croyant doit lire, savoir et appliquer, p. 9.

30. Entretien avec Bamar Gueye, à son bureau, Dakar, le 1er mars 2010.

31. Cette prise de position est loin d'être isolée et spécifique aux musulmans. Les mouvements pentecôtistes sont aussi très réticents devant l'utilisation des préservatifs; voir par exemple le numéro spécial d'Africa Today intitulé « Engaging Christianities: Negotiating HIV/AIDS, Health, and Social Relations in East and Southern Africa », Volume 56, Number 1, Fall 2009 et Pfeiffer (2004a et b).

32. Entretien avec Bamar Gueye, dans son bureau, Dakar, le $1^{\text {er }}$ mars 2010.

33. Catégorie utilisée notamment par l'ONG Jamra.

34. Entretien avec Aïssatou Mbaye, au siège de Jamra, Dakar, le 4 juin 2008.

35. Ancien imâm râtib de la mosquée de l'université Cheikh Anta Diop de Dakar.

36. Cet atelier a eu lieu à l'Institut islamique, à Dakar, les 15, 16 et 17 mars 2010.

37. Cette loi a été votée le 16 février 2010. L'article 13, alinéa 8 du texte de loi stipule: «Les autorités civiles, coutumières et religieuses compétentes en matière matrimoniale encouragent les futures époux à faire le test de dépistage avant la conclusion de leur union ». Dans ce texte, le dépistage est volontaire et non obligatoire alors que certains parlementaires ont émis l'idée d'un dépistage prénatal, prénuptial et d'un dépistage des émigrés et des touristes. De l'avis du ministre Modou Diagne Fada, « les imâms, les évêques et prestataires de services peuvent faire la proposition aux futurs mariés et femmes enceintes » (Wal Fadjri, 17 février 2010, n.p.). Voir aussi certaines critiques émises face à cette loi dans un Rapport d'étude et de diagnostic sur le lien entre l'autonomisation économique et politique des femmes vivant avec le virus du Sida et la loi sur le Vih/Sida » (Niang, Issa, «Sénégal : Loi sur le Vih/Sida-Des insuffisances sur les droits des femmes vivant avec le virus ", in Wal Fadjri, 3 juin 2010, n.p.) 
38. Trinitapoli souligne que les fidèles masculins (sont appelés) à être équitables entre leurs femmes dans un contexte de polygamie (Trinitapoli $2006: 261$ ).

39. Docteur Sakho: "Vous les imâms, c'est vous qui continuez la mission du prophète. Donc nous sommes derrière vous ", atelier, le 15 mars 2010.

40. Qui signifie littéralement « Rien ne vaut le bon voisinage ».

41. Banlieue populaire de Dakar.

42. L'imâm Moussa Gueye parle d'ailleurs d' " école des parents ", entretien avec lui, au siège de Jamra, à Dakar, le 12 juin 2008.

43. Entretien avec imâm Moussa Gueye, au siège de Jamra, le 12 juin 2008.

44. Observation participante, le 10 mars 2010.

45. «Afin de donner un message d'espoir » comme aime à le rappeler Aïssatou Mbaye, dans la cour de l'école, Rufisque.

46. Document interne de présentation de Jamra.

47. Allocution sur les « Enjeux de l'action caritative islamique » de Bamar Gueye.

48. Entretien avec Bamar Gueye, au siège de Jamra, Dakar, le 12 juin 2008.

49. Entretien avec Bamar Gueye, au siège de Jamra, Dakar, le 12 juin 2008. Latif Gueye dirige le parti Le Rassemblement Démocratique Sénégalais (RDS) à partir de mai 2000. «Le 10 décembre 2006, le RDS signe avec le Parti démocratique sénégalais (PDS), un pacte électoral en vue des élections (présidentielle et législatives) de février et juin 2007. Suite à cet accord, Abdou Latif Gueye est investi sur la liste nationale de la Coalition Sopi 2007. Il entre à l'Assemblée nationale où il est élu le 20 juin 2007 vice-président du bureau », Agence de presse sénégalaise, le 6 avril 2008.

50. Entre mai 2000 et octobre 2002, Latif Gueye était conseiller spécial du président Wade, avec rang d'ambassadeur.

51. Accusé de mauvaise gestion et de trafic d'antirétroviraux, Latif Guèye considéra qu'il fut victime d'une «cabale politicienne ». En août 2004, réhabilité par la justice, il put reprendre ses activités.

Ousmane Gueye a la même lecture des faits concernant son différend avec le président de l'association des imâms et des oulémas.

52. Dans le contexte d'une part, de la construction du Monument de la Renaissance, voulue par le président Abdoulaye Wade et vivement décriée par plusieurs composantes de la société civile en raison de son coût et du symbole que la statue représente et d'autre part, dans le contexte de rentrée politique du secrétaire général du RDS, Mame Mactar Guèye, ce parti s'est en effet entredéchiré, voir Georges Nesta Diop, « Sénégal: Imam Massamba Diop, ex-responsable du Rds «Jamais je ne quitterai la famille de Latif Guèye», Wal Fadjri, 22 avril 2010.

53. Créé en 1982, le CONGAD regroupe 178 ONGs nationales, étrangères et internationales. «Le CONGAD a pour vocation de: développer la concertation et les échanges entre les ONG et défendre leurs intérêts ; promouvoir la solidarité inter - ONG dans l'appui aux communautés de base; mobiliser les ONG autour de leurs préoccupations et de leurs besoins en particulier et de ceux de la Société civile en général.

54. Entretien avec Bamar Gueye, à son bureau, Dakar, le $1^{\text {er }}$ mars 2010.

55. Entretien avec Bamar Gueye, au siège de Jamra, Dakar, le 12 juin 2008.

56. Niang, Issa, "Sénégal: 400 cas de viols et d'incestes recensés entre 2006 et 2007 ", Ambenatna.over-blog.com

57. Codou, Bop, «Sénégal : « Homophobie et manipulation politique de l'islam », Women Living Under Muslim Laws Networkers.

58. Bamar Gueye en est secrétaire exécutif.

59. Bamar Gueye en est le secrétaire général adjoint.

60. Bamar Gueye est responsable de la communication dans cette instance.

61. AFP, 29 avril 2009. 
62. C'est l'instance qui prend des positions de nature politique au sein de Jamra.

63. «Mame Mactar Gueye, vice-président de l'ONG Jamra : «Une structure cherche à tromper Wade, pour lui faire signer la pétition légalisant l'homosexualité pour un bonus de 1,5 milliard Cfa », Rewmi.com.

64. Entretien avec Bamar Gueye, à son bureau, Dakar, le $1^{\mathrm{er}}$ mars 2010. Depuis le décès accidentel de Latif Gueye qui était à la fois le président de l'Organisation islamique de Jamra, le président de l'ONG Jamra et le secrétaire général du partir du RDS, les responsabilités ont été réparties comme suit : l'imâm Massamba Diop a été désigné comme le président de l'organisation islamique Jamra et Bamar Gueye est devenu le directeur exécutif de l'ONG.

65. Doucouré, A. «Sénégal: Après la sortie de Wade les traitant « d'Ignorants » - Les imams ripostent ", Sud quotidien, 11 décembre 2009.

\section{RÉSUMÉS}

Cette contribution invite à explorer comment deux structures, l'ONG Jamra et l'association Réseau national Islam Sida Éducation, participent à la lutte contre le Sida au Sénégal et deviennent à la fois des relais incontournables de la politique étatique et des bailleurs de fonds internationaux mais aussi des partenaires critiques voire indociles. Cela permet à la fois de démontrer l'ambivalence des relations entre ONG et État qui oscillent entre complémentarité et concurrence et d'analyser le jeu de négociations qui s'opère à travers la dialectique entre les fondements éthiques de l'islam, la collaboration avec divers partenaires (État, bailleurs de fonds etc.) et l'arrimage aux programmes d'aide au développement international. Le dispositif d'actions mis en place par ces deux structures rend compte, par ailleurs, de formes originales de participation citoyenne qui semblent préfigurer de l'émergence de modes populaires d'action volontariste.

This article analyzes to which extent two organizations -the NGO Jamra and the Réseau national Islam Sida Éducation- participate in the battle against AIDS in Senega, and become essential agents of state politics, international donors, and also critical or even indocile partners. This situation allows us to highlight the ambiguous relationship between NGO and State, which oscillates between complementarity and competition, and the complex system of negotiations that is caused by the dialectical relationship between the ethical foundations of Islam, the collaboration between different partners (State, international donors, etc.), and the interconnections between programmes that further international development. The system of actions put into place by these two organizations makes obvious, among other things, original forms of the citizen participation that appear to foreshadow the emergence of a popular form of voluntarist action. 